\title{
Smart-RF for mmWave MIMO Beamforming
}

\author{
Praneeth Susarla, Jani Saloranta, Giuseppe Destino, Olli Kursu, \\ Marko Sonkki, Marko E. Leinonen, Aarno Pärssinen \\ University of Oulu, Oulu, Finland \\ email: firstname.lastnamedoulu.fi
}

\begin{abstract}
We study the performance of a prototype of a millimeter-wave transceiver with radio frequency (RF) beamforming capabilities. More specifically, the focus is on the architecture and software implementation of a smartRF that allows self-beam-alignment. Simulation studies shows the expected performance in terms of probability of misalignment and coverage using different beam search strategies as well as realistic antenna beam patterns and phase-shifters quantization constraints.
\end{abstract}

\section{INTRODUCTION}

Millimeter-wave communication is considered one of the key innovations of $5^{\text {th }}$ Generation (5G) networks, to enable tens of Gbps data rate as well as massive multiple-input-multiple-output (MIMO) and beamforming [1]-[3]. However, many challenges for hardware implementation, signal processing and algorithms are yet to be solved.

The EU-KR H2020 5GCHAMPION project [4] is, for instance, one of the first research projects delivering a prototype of millimeter wave $(\mathrm{mmW})$ transceiver at $28 \mathrm{GHz}$, integrated with pre-commercial $5 \mathrm{G}$ base-band and core networks components. One big challenge addressed in the project, especially considering hardware constraints, is the initial access procedure in which both transmitter and receiver need to establish a reliable radiolink connection.

Most of the previous works [5]-[7] in mmW MIMO beamforming have been mainly on different beam searching strategies, while some focused on implementing novel schemes for aligning multiple beams [8]-[10] in terms of maximum signal power, by exploiting the directionality of $\mathrm{mmW}$ communications.

Our objective through this paper, is to provide an efficient and flexible mechanism to develop as well as test different beam training and alignment algorithms on real RF hardware units through Micro-Controller Unit (MCU). We propose self-beam-alignment mechanisms implemented in the RF unit and study the coverage and probability of beam alignment with different beam search strategies. In this regard, we also focus on implementation aspects of the digital control hardware and software architecture for smart-RF.

The remainder of the paper is organized as follows. In Section II, we propose the principles of our smart-RF design of control board. In Section III, the focus is on the software architecture for RF beamforming. Details on the beamforming codebook for self-beam-alignment are also provided in III-A. Section IV is dedicated to self-beam-alignment procedure and in Section $\mathrm{V}$, we show simulation results on RF beam control using quantized phase shifter levels, real antenna beam patterns and different search strategies. Finally, in Section VI, concluding remarks are provided.

\section{OVERVIEW OF A SMART-RF IMPLEMENTATION}

The characterization and usage of a backhaul transceiver radio unit have been presented in [11]. The antenna unit includes two similar radio boards with RF beamforming and phased-array antennas and one AUXiliary/Control (AUX) board to manage the functionalities of the beamformer. The beamformer is implemented with phase-shifters at mmW level using 5bit phase quantization. A three-state switch is added in each transmission line to set each antenna port in either transmit or receive or idle state.

In full operational mode two antenna units are used, each with more than $60 \mathrm{dBm}$ Effective Isotropic Radiated Power (EIRP). The RF units are connected to a Digital Front-End (DFE), providing 8 channels with $400 \mathrm{MHz}$ signal bandwidth transmission. The achievable data-rate along with the expected link-budget with different modulation scheme are provided in [12], [13].

A block diagram in Fig. 1 presents the main system blocks and interfaces of the full $28 \mathrm{GHz}$ backhaul unit.

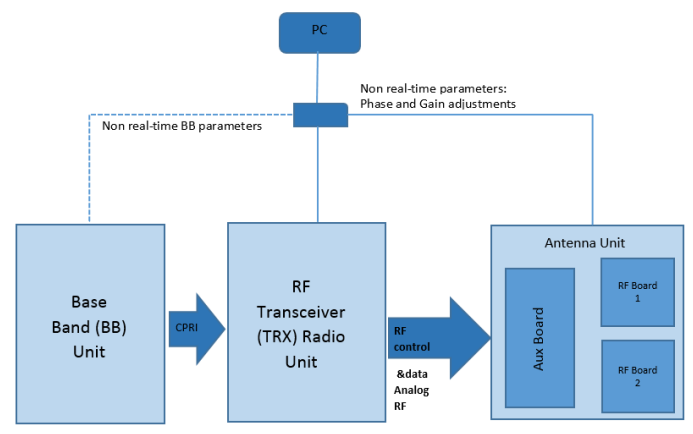

Figure 1. 5GCHAMPION main system blocks [13].

Hereafter, our focus is on the radio unit and more specifically on the AUX board which is equipped with MCU, i.e., ST Nucleo processor, and several analog and digital interfaces enabling control functionalities of the 


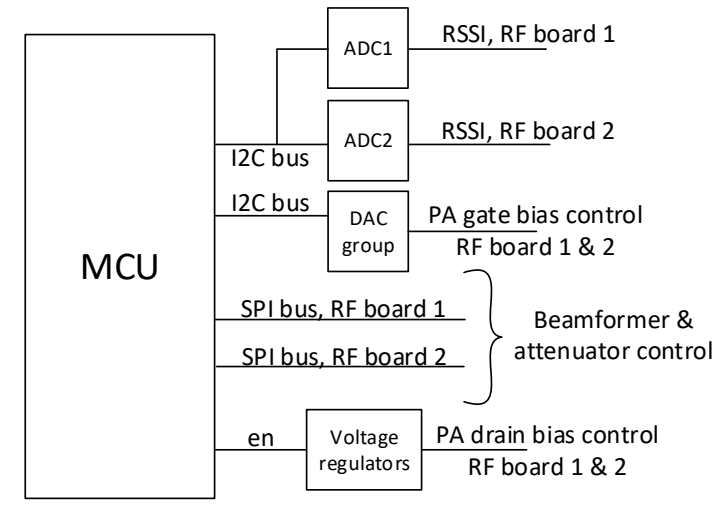

Figure 2. Control Board design of smart-RF

RF components including phase-shifters, TX and RX attenuators, Power Amplifier (PA) bias control, Received Signal Strength (RSS) measurements etc. as shown in Fig 2. In addition to direct $\mathrm{I} / \mathrm{O}$ direct controls, two SPI buses and two I2C buses are used to control RF boards. SPI buses control Local Oscillator (LO) generation, phase-shifter settings and $\mathrm{mmW}$ signal attenuators while I2C buses multiple devices especially PA gate bias programming for both RF boards as seen in Fig 2.

The MCU is synchronized with the DFE via timing signals that indicate the TX/RX slot as well as radioframe start (10 ms periodic signal). These signals are key for managing the self-beam-alignment procedure.

In the receiving path, a specific power reading block is used to measure the receiving signal power after the beamformer distribution line. More specifically, this block consists of analog-to-digital conversion (ADC) converting the receiving signal voltage to signal power. Details of the power detector and interface to the MCU are provided in D3.1 [13].

\section{BEAMFORMER CONTROL SOFTWARE ARCHITECTURE}

The beamformer software is implemented with the layered architecture as shown in Fig 3. The architecture is implemented in four layers namely, Application, Middleware, Hardware Abstraction Layer (HAL) and Driver level layers. The communication of application and middleware with lower layers happens through Ethernet and Message Passing Interface (MPI).

The driver layer deals with logical phase-shifters and a trigger pulse. Phase-shifter registers are loaded and latched to the phase-shifters upon a trigger pulse that is synchronized with a radio frame start pulse. Reading Received Signal Strength Index (RSSI) ADC voltages from the RF boards and usage of Electrically Erasable Programmable Read-Only Memory (EEPROM) for storing them are dealt at driver level, in order to help computing RSSI power measurements in higher layers. Besides, digital-to-analog Converter (DAC)'s which are

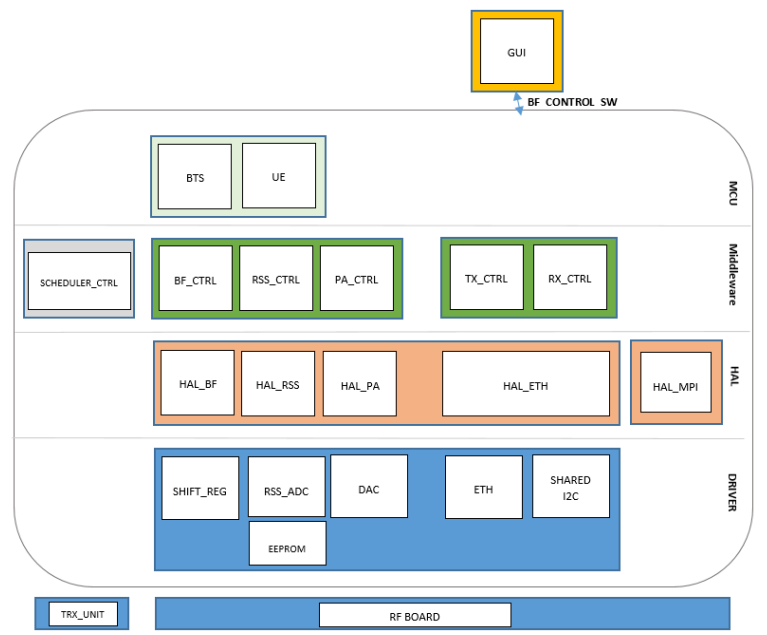

Figure 3. Beamformer control software architecture

useful for PA voltage control and Low Noise Amplifier (LNA) enable/disable, are also dealt in this layer.

HAL contains a library of functionalities to control the drivers in the lower layer. At the HAL, the beam direction is taken as input from above layers and converted into respective beamforming weights under $H A L \_B F$. HAL_RSS deals with converting received RSSI ADC voltages from lower layer to power and sending them to above layers. $H A L_{-} P A$ deals with handling enabling/disabling, calibrating and saving the voltage values received from the above layers during beamforming setup and sending them appropriately to PA gate bias control DACs in lower layers. HAL_ETH and HAL_MPI helps in providing functionalities for setting up Ethernet and MPI communication interfaces between higher and lower layers.

The middleware comprises of all control threads, which will run simultaneously, communicate via MPI and use HAL library. At the middleware, reading of RSSI values from the hardware is instantiated and held in Idle state unless it receives a signal from the above layer. On receiving the signal, the middleware then proceeds through beam direction assessment, by computing the beam direction with maximum signal-tonoise ratio (SNR), based on RSSI power measurements. The application layer deals with instantiating the beam alignment procedure and triggers a signal at lower layers depending on the need to align the RF beams.

\section{A. Beamforming Solution}

We have implemented three different beamforming methods to demonstrate the possibilities of smart-RF MIMO beamforming, using the control software mentioned in Section III. We followed Exhaustive as well as Hierarchical codebook design strategy with different beam widths [5], to achieve better search efficiency on 
beam alignment techniques between Transmitter Radio Unit (TRU) and Receiver Radio Unit (RRU).

NARROW: This approach performs an exhaustive search for beam sweeping and then align them using uniform DFT codebooks. This method greatly limits the usage of more number of antennas at both transmitter and receiver as it could make beam training computationally expensive.

DEACT: This is a brute-force antenna deactivation method, using a binary tree hierarchical codebook, where the beams get generated by turning off part of the antennas. This hierarchical search approach is much more time efficient than the above exhaustive search methodology. Although, its theoretical and simulation results are better than state-of-art techniques as mentioned in [14], the method greatly limits the maximal total transmission power of an $\mathrm{mmW}$ device.

SUBARRAY: This approach is based on the subarray broadening deactivation technique using hierarchical codebook design, proposed in [5]. In this method, all the codewords either have all antennas activated or half of them activated, showing significant advantage over $D E A C T$ method in terms of maximal total transmission power.

A control board associated with real boards can be helpful in emulating the various beamforming results using above different methods on radio unit hardware. It can also help with beamforming using a joint combination of these approaches (e.g. a joint SUBARRAY and a $D E A C T$ approach [5]) for different scenarios in the future.

\section{TECHNIQUE FOR SELF-BEAM ALIGNMENT}

The beam alignment procedure, hereafter also referred to as beam training, is designed for pairing a transmit and a receiving beam. Also, it is computationally expensive when more antenna elements are involved and also occurs prior communication. In short, the objective is to align TRU and RRU beams so as to maximize the SNR for data transmission.

The MCU is responsible of the beam-adjustment procedure and the state diagram shown in Fig. 4 describes the sequence of functions applied during this process. The whole beamtraining process is periodically repeated, where the period is determined by an estimate of the mobility.

In this paper, two possible strategies for beam alignment, namely, exhaustive and hierarchical search [15] are considered. The former consists of a technique where both TRU and RRU sweeps the whole beam codebook (DFT-based) to detect the pair yielding the maximum energy. The latter is a more time efficient approach as TRU and RRU start with a set of broad beams and based

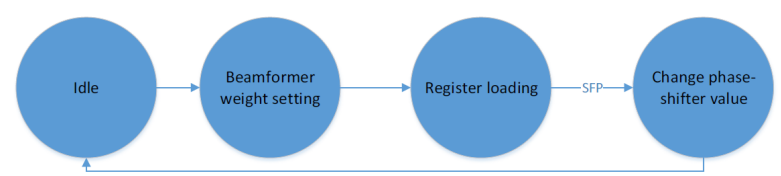

Figure 4. Beam Adjustment State Diagram.

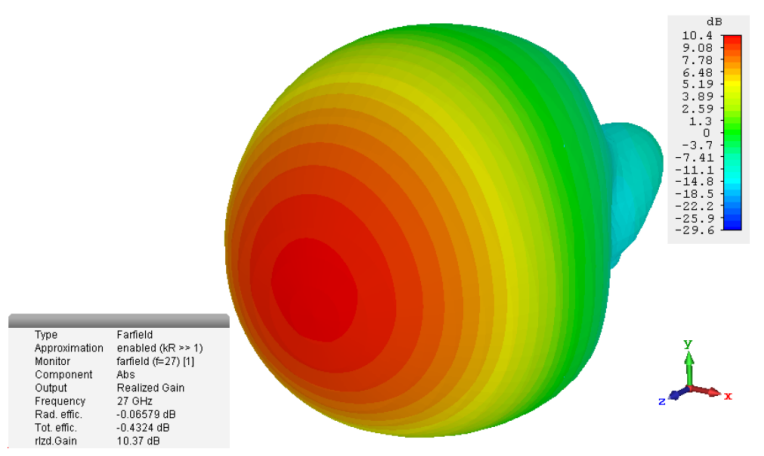

Figure 5. Simulated antenna (2x2 sub-array) element 3D RF beampattern at $27 \mathrm{GHz}$ [16]

on feedback signal power reporting ${ }^{1}$, move forward with a set of narrower beams.

In order to simulate the expected behavior of the beam-alignment procedure, we model the communication between TRU and RRU as follows. The radio channel is a single ray link (dominant line-of-sight (LOS)) with propagation delay $\tau$, angle-of-arrival $\theta$ and angle-of-departure $\phi$. Mathematically, we define a matrix $\mathbf{H} \in \mathbb{C}^{N \times N}$ as

$$
\mathbf{H}(t ; \tau, \theta, \phi, \rho) \triangleq c \mathbf{a}_{R}(\theta) \mathbf{a}_{T}^{H}(\phi) \delta(t-\tau),
$$

where $c \sim \mathcal{C N}\left(0, \sigma^{2} \mathbf{I}\right)$, with $\mathcal{C N}$ indicating complex guassian distribution, $\sigma^{2}$ is the variance proportional to the path-loss and $\mathbf{a}_{R}(\cdot)$ and $\mathbf{a}_{T}(\cdot)$ are the receiving and transmit steering vector, respectively.

The steering vectors includes both array factor as well as real element beampattern gain, i.e.,

$$
\mathbf{a}_{R}(\theta)=\mathbf{a}(\theta) \psi(\theta),
$$

where

$$
\mathbf{a}(\theta)=\left[1, \cdot, e^{j 2 \pi \lambda d(N-1) \sin (\theta)}\right],
$$

where $d=\lambda$ is the space between the antenna elements formed by $2 \times 2$ sub-array with beampattern shown in Fig. 5.

The received signal captured at the output of the beamformer is given by

$$
y(t)=\mathbf{w}^{\mathrm{H}} \mathbf{H} \mathbf{f} p(t-\tau)+\mathbf{n}(t),
$$

where $p(t)$ is the transmitted waveforms of duration $T_{s}$, periodicity $T_{p}$, and power $P=\frac{1}{T_{s}} \int_{0}^{T_{s}}\|p(t)\|^{2} d t$.

${ }^{1}$ Feedback channel is assumed available via side-communication links for control messages. 


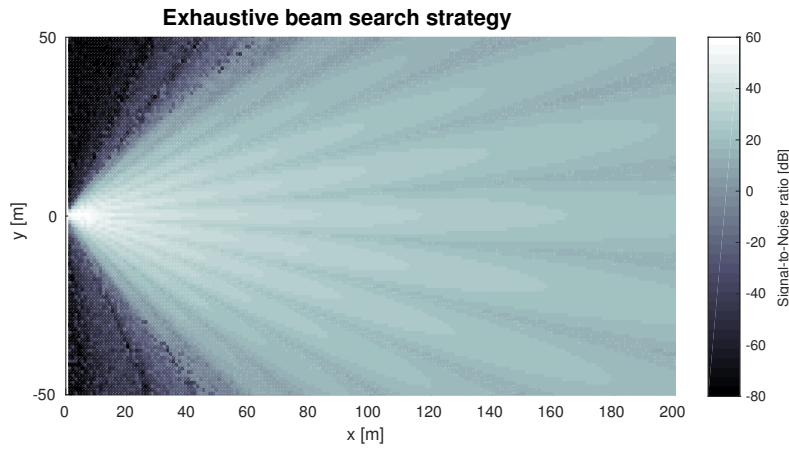

(a) RRU SNR levels using exhaustive beam search strategy.

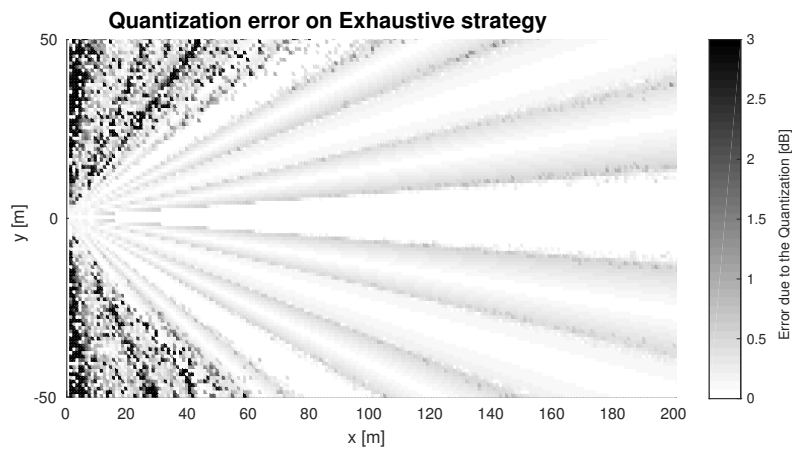

(b) The magnitude of an error due to the quantization of phase shifter values on Nucleo MCU in exhaustive beam search algorithm.

Figure 6. The performance of the exhaustive search strategy.

The log-power detector output used to measure the output power of the receiving RF path is modeled with a signal $v(t)$ that is proportional ${ }^{2}$ to the instantaneous power $s(t)$

$$
s(t)=10 \log _{10}\left(\frac{1}{T_{d}} \int_{t}^{t+T_{d}}\left\|y_{i}(t)\right\|^{2} d t\right),
$$

where $T_{d}$ is the integration time tunable by a capacitor.

At the micro-controller, the power $p(t)$ is sampled and averaged over the duration of the waveform pulse to provide an estimate of the received signal strength.

\section{Simulation Study}

We consider an MIMO-orthogonal frequency-division multiplexing (OFDM) communication system, similar to [15], [17] and look into two beam alignment strategies as mentioned in Section IV, namely, exhaustive search and hierarchical search strategy. The transmit signal is OFDM with 2048 sub-carriers of which $\mathrm{N}_{\mathrm{FFT}}=1200$ sub-carriers in use with $\Delta_{f}=75 \mathrm{kHz}$ spacing, center frequency is selected $f_{c}=28 \mathrm{GHz}$. Transmit power is $P_{t x}=10 \mathrm{dBm}$ and TRU is located at $[0,0]$. The TRU and the RRU has same number of antennas elements $N=8$ ( 1 element consists of $2 \times 2$ subarray), as studied in 5GCHAMPION proof-of-concept demonstration platform [4].

\footnotetext{
${ }^{2}$ Note that proportionality holds only in the linear region of the power detector.
}

We investigate the effect of quantization of phasevalues, namely each phase-value for beamformer weights are set by 5-bit on MCU hardware and compared with optimal float values. The simulations were carried out in scenario of $100 \mathrm{~m} \times 200 \mathrm{~m}$ region with TRU located at $(0,0)$. The TRU is aligned so that antenna boresight is facing direction of $0^{\circ}$ while RRU is always facing $180^{\circ}$.

Fig. 6 illustrates the performance of the exhaustive search strategy. Firstly, in Fig. 6a it can be noted that SNR drops significantly outside of the boresight region $\left[+30^{\circ} \ldots-30^{\circ}\right]$. This is due to the antenna radiation pattern presented in Fig. 5. Secondly, it can be noted that when using a fixed codebook, the directions are clearly visible where small change in angular domain causes a drop in SNR.

The error caused by quantization of phase shifter values set on Nucleo MCU is illustrated in Fig. 6b. It can be observed that on aforementioned region $\left[+30^{\circ} \ldots-30^{\circ}\right]$ where antenna is designed to operate, the drop in performance due to the quantized phase shifter values is less than $0.5 \mathrm{~dB}$ and in angular domain $\left[+60^{\circ} \ldots-60^{\circ}\right]$ less than $3 \mathrm{~dB}$. Also, less number of quantization errors are observed within the boresight region, with major errors among them witnessed at particular azimuthal angles with respect to $(0,0)$. As a result, drop in SNR is observed to be fairly low throughout the area within aforementioned region, thus exhibiting a good coverage.

Fig. 7 shows similar simulation cases for hierarchical beam search strategy with significant drop in SNR values outside the boresight region as well as change in angular domains within visible region through fixed beamforming codebook. The main difference between hierarchical and exhaustive search strategy is that in hierarchical strategy, wider beamwidth is used to transmit (and receive) in its first levels.

Besides, it is more time efficient by reducing the number of cycles in search procedure (compared to exhaustive) as it is not necessary to examine all possible combination of beam directions. However, we note from Fig. 7a that the overall performance with respect to SNR in hierarchical search strategy is slightly worse than with exhaustive search strategy - as expected. That is because if error on beam selection is done in level $k$ in hierarchical search, it is not possible to realign the beam search procedure to capture the correct beam on levels $k+1$ [17].

Secondly, as seen in Fig. 7b, the drop in SNR is fairly low until $120 \mathrm{~m}$ (along the $x$-axis) and the signal deteriorates beyond that, exhibiting less coverage relative to that observed in Fig. 6b. Thus, it proves that hierarchical search offers less coverage compared to exhaustive strategy under similar conditions. This is aligning with its theoretical principles. Also, majority of the large quantization errors are witnessed after $120 \mathrm{~m}$ along the $x$ axis inside the boresight region and in between $60-120 \mathrm{~m}$ 


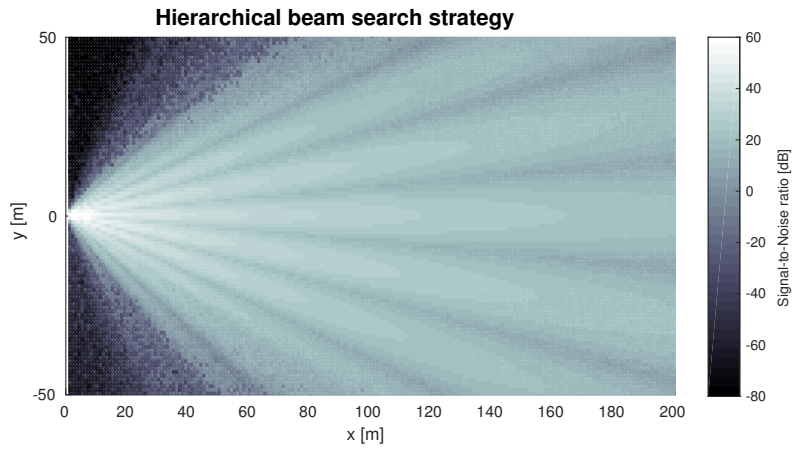

(a) RRU SNR levels using hierarchical beam search strategy.

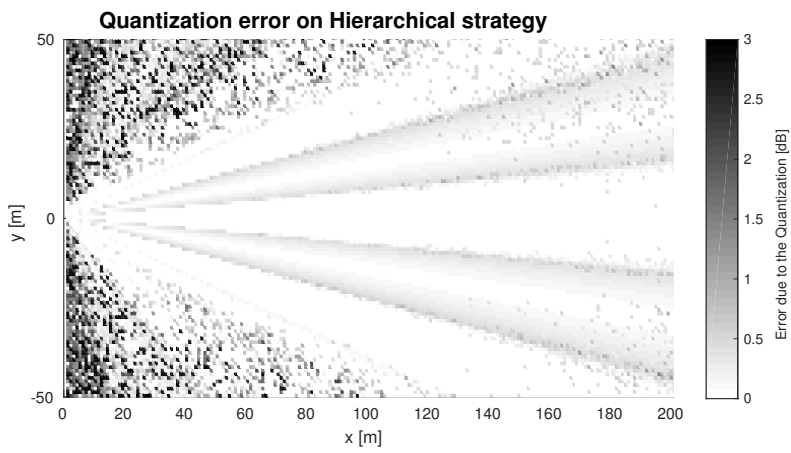

(b) The magnitude of an error due to the quantization of phase shifter values on Nucleo MCU in hierarchical beam search algorithm.

Figure 7. The performance of the hierarchical search strategy.

along $x$-axis at extremities of the region compared to errors observed in Fig. 6b. This shows that quantization effects are realized more in hierarchical beam search strategy than in exhaustive strategy. This is due to the fact that hierarchical strategy changes its codebook of beam directions while scanning through its different levels.

We also investigate the performance of both beam alignment strategies through their probabilities of misalignment as shown in Fig. 8. We evaluate the percentage of probabilities of beam alignments at different azimuthal angles with respect to the antenna boresight, by increasing the distance between TRU and RRU units for both beam alignment strategies.

From Fig.8 we notice that the beamalignment for longer distances between TRU and RRU units is better in exhaustive search compared to hierarchical one. The reason is two fold, first, the hierarchical search strategy has more possibilities to make errors while performing beam scanning on different levels, and this is especially realized on low SNR regime (longer distances between RRU and TRU), and second, the existence of slightly higher phase-quantization errors in hierarchical compared to exhaustive search as observed in Fig. $6 \mathrm{~b}$ and Fig. $7 b$.

Also, the probability of beam-alignment decreases with increase of azimuth angles with respect to $x$-axis of the boresight region, within exhaustive search method

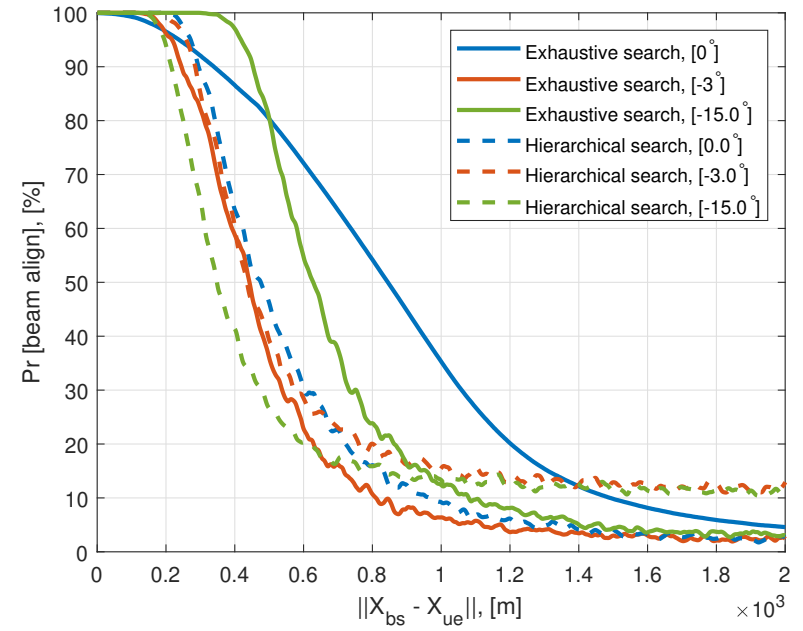

Figure 8. Probability of alignment during beam adjustment in exhaustive (solid lines) and hierarchical (dashed lines) search strategies in three fixed angular values namely, $0^{\circ},-3^{\circ}$ and $-15^{\circ}$.

unlike the opposite way is observed in hierarchical search. The reason is the realistic antenna patterns used in the simulation study. The direction $0^{\circ}$ is with the strongest antenna gain factor.

To conclude, we note that the above beamalignment results obtained from beamformer control software developed for 5GCHAMPION antenna units comply well with theoretical explanations between both the exhaustive and hierarchical beam strategies [18]. Furthermore, the implemented software architecture described above helps in efficient beamforming testing.

\section{CONCLUSION}

As discussed in the paper, a control unit with RF boards could be highly useful in faster prototyping of different RF beamforming methods in the real environment. It provides flexibility in designing, implementing and analyzing (e.g. different codebook designs for beam searching), beam alignment solutions using sampling frequency, memory buffers and measurements of RSS signals. For example, based on the analysis witnessed under section $\mathrm{V}$, antenna units can automatically switch to exhaustive strategy when observed SNR levels are low and to hierarchical search when the SNR levels are high as it reduces the overall beam training process. It can also be helpful in automatic real-time adjustments of RF beam weight vectors using machine learning, during misalignments in future.

\section{ACKNOWLEDGMENTS}

The research leading to these results has received funding from the European Union H2020 5GPPP under grant no. 723247 and supported by the Institute for Information \& communications Technology Promotion (IITP) grant funded by the Korea government (MSIP) (No.B0115-16-0001, 5GCHAMPION). 


\section{REFERENCES}

[1] Z. Pi and F. Khan, "An introduction to millimeter-wave mobile broadband systems," IEEE Commun. Mag., vol. 49, no. 6, pp. 101-107, Jun. 2011.

[2] P. Wang, Y. Li, L. Song, and B. Vucetic, "Multi-gigabit millimeter wave wireless communications for $5 \mathrm{~g}$ : from fixed access to cellular networks," IEEE Commun. Mag., vol. 53, no. 1, pp. 168-178, Jan. 2015.

[3] R. W. Heath, N. González-Prelcic, S. Rangan, W. Roh, and A. M. Sayeed, "An Overview of Signal Processing Techniques for Millimeter Wave MIMO Systems," IEEE J. Sel. Topics Signal Process., vol. 10, no. 3, pp. 436-453, Apr. 2016.

[4] M. Mueck et al., "5G CHAMPION - Rolling out 5G in 2018," in Proc. IEEE Global Commun. Conf. Workshops, Dec. 2016, pp. $1-6$.

[5] Z. Xiao, T. He, P. Xia, and X. G. Xia, "Hierarchical codebook design for beamforming training in millimeter-wave communication," IEEE Trans. Wireless Commun., vol. 15, no. 5, pp. 33803392, May 2016.

[6] C. Liu, M. Li, I. B. Collings, S. V. Hanly, and P. Whiting, "Design and analysis of transmit beamforming for millimeter wave base station discovery," IEEE Transactions on Wireless Communications, vol. 16, no. 2, pp. 797-811, 2017.

[7] Z. Xiao, P. Xia, and X.-G. Xia, "Codebook design for millimeterwave channel estimation with hybrid precoding structure," IEEE Transactions on Wireless Communications, vol. 16, no. 1, pp. 141-153, 2017.

[8] S. Sun and T. S. Rappaport, "Multi-beam antenna combining for $28 \mathrm{ghz}$ cellular link improvement in urban environments," in Global Communications Conference (GLOBECOM), 2013 IEEE. IEEE, 2013, pp. 3754-3759.

[9] S. Sun, G. R. MacCartney, M. K. Samimi, S. Nie, and T. S. Rappaport, "Millimeter wave multi-beam antenna combining for $5 \mathrm{~g}$ cellular link improvement in new york city," in Communications (ICC), 2014 IEEE International Conference on. IEEE, 2014, pp. $5468-5473$.

[10] B. W. Ku, D. G. Han, and Y. S. Cho, "Efficient beam-training technique for millimeter-wave cellular communications," ETRI Journal, vol. 38, no. 1, pp. 81-89, 2016.

[11] G. Destino, O. Kursu, S. Tammelin, J. Haukipuro, M. Sonkki, T. Rahkonen, A. Pärssinen, M. Latva-aho, A. Korvala, and M. Pettissalo, "System Analysis and Design of mmW Mobile Backhaul Transceiver at $28 \mathrm{GHz}$," in Proc. Eur. Conf. Networks and Commin., Jun. 2017.

[12] M. E. Leinonen, G. Destino, O. Kursu, M. Sonkki, and A. Pärssinen, "28 ghz wireless backhaul transceiver characterization and radio link budget," ETRI Journal, vol. 40, no. 1, pp. 89-100, 2018.

[13] A. Pärssinen et al., "Deliverable 3.1: Front-End Design," in 5GCHAMPION Project, Nov. 2016.

[14] T. He and Z. Xiao, "Suboptimal beam search algorithm and codebook design for millimeter-wave communications," Mobile Networks and Applications, vol. 20, no. 1, pp. 86-97, 2015.

[15] J. Saloranta, G. Destino, and H. Wymeersch, "Comparison of Different Beamtraining Strategies from a Rate-Positioning TradeOff Perspective," in Proc. Eur. Conf. Networks and Commin., 2017.

[16] M. Sonkki et al., "Linearly polarized 64-element antenna array for mm-wave mobile backhaul application," EuCAP, 2018.

[17] G. Destino, J. Saloranta, H. Wymeersch, and G. Seco-Granados, "Impact of Imperfect Beam Alignment on the Rate-Positioning Trade-Off," in Proc. IEEE Wireless Commun. and Networking Conf.: Special Session Workshops (WCNC2018-SSW), Apr. 2018.

[18] V. Desai, L. Krzymien, P. Sartori, W. Xiao, A. Soong, and A. Alkhateeb, "Initial beamforming for mmwave communications," in Signals, Systems and Computers, 2014 48th Asilomar Conference on. IEEE, 2014, pp. 1926-1930. 